\title{
Randomized phase II trial of a first-in-human cancer cell lysate vaccine in patients with thoracic malignancies
}

\author{
Mary Zhang ${ }^{1 \#}$, Julie A. Hong ${ }^{1 \#}$, Tricia F. Kunst ${ }^{1}$, Colleen D. Bond ${ }^{1}$, Cara M. Kenney ${ }^{1}$, Cheryl L. Warga ${ }^{1}$, \\ Javier Yeray ${ }^{2}$, Min-Jung Lee ${ }^{3}$, Akira Yuno ${ }^{3 *}$, Sunmin Lee ${ }^{3}$, Markku Miettinen ${ }^{4}$, R. Taylor Ripley ${ }^{1 *}$, \\ Chuong D. Hoang ${ }^{1}$, Sacha Gnjatic ${ }^{2}$, Jane B. Trepel ${ }^{3}$, David S. Schrump ${ }^{1}$ \\ ${ }^{1}$ Thoracic Epigenetics Section, Thoracic Surgery Branch, Center for Cancer Research, National Cancer Institute, Bethesda, MD, USA; ${ }^{2}$ Tisch \\ Cancer Institute, Icahn School of Medicine at Mount Sinai, New York, NY, USA; ${ }^{3}$ Developmental Therapeutics Branch, Center for Cancer \\ Research, National Cancer Institute, Bethesda, MD, USA; ${ }^{4}$ Laboratory of Pathology, Center for Cancer Research, National Cancer Institute, \\ Bethesda, MD, USA \\ Contributions: (I) Conception and design: S Gnjatic, JB Trepel, DS Schrump; (II) Administrative support: M Zhang, JA Hong; (III) Provision of study \\ materials or patients: TF Kunst, CD Bond, CM Kenney, CL Warga, RT Ripley, CD Hoang, DS Schrump; (IV) Collection and assembly of data: \\ TF Kunst, CM Kenney, J Yeray, MJ Lee, A Yuno, S Lee, M Miettinen; (V) Data analysis and interpretation: S Gnjatic, JB Trepel, DS Schrump; (VI) \\ Manuscript writing: All authors; (VII) Final approval of manuscript: All authors. \\ "These authors contributed equally to this work. \\ Correspondence to: David S. Schrump, MD, MBA. Thoracic Surgery Branch, Center for Cancer Research, NCI, Building 10, 4-3942, 10 Center Drive, \\ Bethesda, MD 20892, USA. Email: David_Schrump@nih.gov.
}

Background: Although most malignancies express cancer-testis antigens (CTA), immune responses to these proteins are limited in thoracic oncology patients. This trial was undertaken to examine if a cancer cell lysate vaccine could induce immunity to CTA, and to ascertain if metronomic cyclophosphamide and celecoxib enhances vaccine-induced immune responses.

Methods: Eleven patients with primary thoracic malignancies and 10 patients with extrathoracic neoplasms metastatic to the chest rendered NED by conventional therapies were randomized to receive H1299 lung cancer cell lysates $\left(10 \mathrm{mg}\right.$ protein/vaccine) with Iscomatrix ${ }^{\mathrm{TM}}$ adjuvant via deep intradermal injection $\mathrm{q} 4$ weeks $\times 6$ with or without daily oral metronomic cyclophosphamide/celecoxib. The primary endpoint was serologic response to purified CTA assessed 1 month after the $6^{\text {th }}$ vaccination. Secondary endpoints included assessment of the effects of cyclophosphamide and celecoxib on frequency and magnitude of vaccine-induced immune responses to CTA. Exploratory endpoints included evaluation of the effects of the vaccine regimens on peripheral immune subsets. Standard of care imaging studies were obtained at baseline and 1 month after the $3^{\text {rd }}$ and $6^{\text {th }}$ vaccinations.

Results: All patients exhibited local and systemic inflammatory responses lasting 72-96 hours following vaccinations. There were no dose limiting treatment related toxicities. Fourteen patients (67\%) completed all six vaccinations. Eight of 14 patients (57\%) exhibited serologic responses to NY-ESO-1. One patient developed antibodies to GAGE7; several patients exhibited reactivity to XAGE and MAGE-C2. Vaccine therapy decreased the percent of Tregs $(\mathrm{P}=0.0068)$, PD-1 expression on Tregs $(\mathrm{P}=0.0027)$, PD-L1 expression on $\mathrm{CD}_{14}{ }^{+}$monocytes $(\mathrm{P}=0.0089)$, PD-L1 expression on classical monocytes $(\mathrm{P}=0.016)$, and PD-L1 expression on intermediate monocytes $(\mathrm{P}=0.0031)$. Cyclophosphamide/celecoxib did not appear to increase immune responses or enhance vaccine-induced alterations in peripheral immune subsets.

Conclusions: H1299 lysate vaccines with Iscomatrix ${ }^{\mathrm{TM}}$ induce immune responses to CTA and modulate peripheral immune subsets in a manner that may enhance antitumor immunity in patients with thoracic malignancies.

Keywords: Vaccine; first-in-human; cancer-testis antigen; thoracic malignancy; pulmonary metastasis

\footnotetext{
* Current address of Akira Yuno: Kumamoto University Hospital, Kumamoto, Japan; R. Taylor Ripley: Baylor College of Medicine, Houston, TX, USA.
} 
Submitted Jan 01, 2021. Accepted for publication May 21, 2021.

doi: $10.21037 /$ tlcr-21-1

View this article at: https://dx.doi.org/10.21037/tlcr-21-1

\section{Introduction}

Identification of serologic and cell mediated immune responses to shared as well as unique (neo) antigens on tumor cells, and impressive, durable responses following immune checkpoint blockade in cancer patients have provided unequivocal evidence supporting the theories of immune surveillance and immunoediting articulated by Burnet, and Schreiber, respectively $(1,2)$. Transformed cells that escape elimination by immune surveillance establish a dynamic equilibrium with the host immune system. Continued selection pressure on cancer cells and the tumor microenvironment ultimately promotes "escape" of immuno-edited clonal variants and evolution of central as well as peripheral immune tolerance resulting in clinically evident malignancies (2).

Genome-wide DNA demethylation during malignant transformation leads to de-repression of a variety of genes that normally exhibit stage-specific expression during germ cell development in immunoprivileged testes or ovary (3). Aberrant activation of these cancer-testis (CT) genes (also referred to as cancer-germline genes) in somatic cells results in expression of highly restricted tumor-associated antigens that induce serologic as well as cell-mediated immune responses in cancer patients; as such, cancer-testis antigens (CTAs) have emerged as attractive targets for cancer immunotherapy (4). To date, over 200 CTAs have been identified, approximately half of which are encoded on the $\mathrm{X}$ chromosome (http://www.cta.lncc.br/). Relative to autosomal CT genes, CT-X chromosome genes often comprise extended families and are more frequently activated in cancer cells; particular gene families appear to be coordinately derepressed in a tumor-specific manner suggesting functional oncogenic interactions (5). To date, cellular immune responses have been detected against more than 20 CT-X antigens in patients with various solid tumors (6).

Whereas most human malignancies express CT-X antigens, immune responses to these proteins are often limited in cancer patients due to low-level, heterogeneous antigen expression, epigenetic repression of genes regulating antigen processing and presentation, and local as well as systemic immunosuppression $(4,7)$. Previously we demonstrated that epigenetic agents such as Decitabine,
Romidepsin, and DZNep can up-regulate CT-X antigen expression in thoracic malignancies (but not normal cells) in vitro and in vivo rendering them susceptible to lysis by cytotoxic T lymphocytes (CTL) recognizing NY-ESO-1 and MAGE-A3 (8-12). Furthermore, we have demonstrated that systemic administration of Decitabine followed by adoptive transfer of CTL recognizing the CTA P1A eradicates pulmonary metastases in an immunocompetent murine model (13). These observations, together with recent reports demonstrating high level CT-X gene expression in cancer stem cells (14-16), raise the possibility that pharmacologic interventions that enhance expression and presentation of CTA-particularly when administered in the context of a primed immune system may be novel strategies to eradicate dormant cancer stem cells that give rise to local as well as systemic recurrences in patients following potentially curative interventions. Herein we report results of a First-in-Human trial evaluating if a lung cancer lysate vaccine administered with or without metronomic oral cyclophosphamide and celecoxib as a strategy to inhibit suppressive activity of regulatory $\mathrm{T}$ (Treg) cells can induce immunity to potentially relevant CTA in patients with thoracic malignancies. We present this article in accordance with the CONSORT reporting checklist (available at https://dx.doi.org/10.21037/tlcr-21-1).

\section{Methods}

\section{Ethical statement}

The trial was conducted in accordance with the Declaration of Helsinki (as revised in 2013) and the guidelines for Good Clinical Practice. The trial (NCT02054104) was approved by NCI-IRB and FDA (IND 15812), and written informed consent was obtained from all individual participants.

\section{Patients and protocol eligibility}

Patients 18 years or older with ECOG performance status of 0-2 who had no clinical evidence of active disease (NED) detected by physical exam, CT scans of chest, abdomen, and pelvis, fused torso and if clinically indicated extremity PET/CT scans, and brain MR scans following standard of 


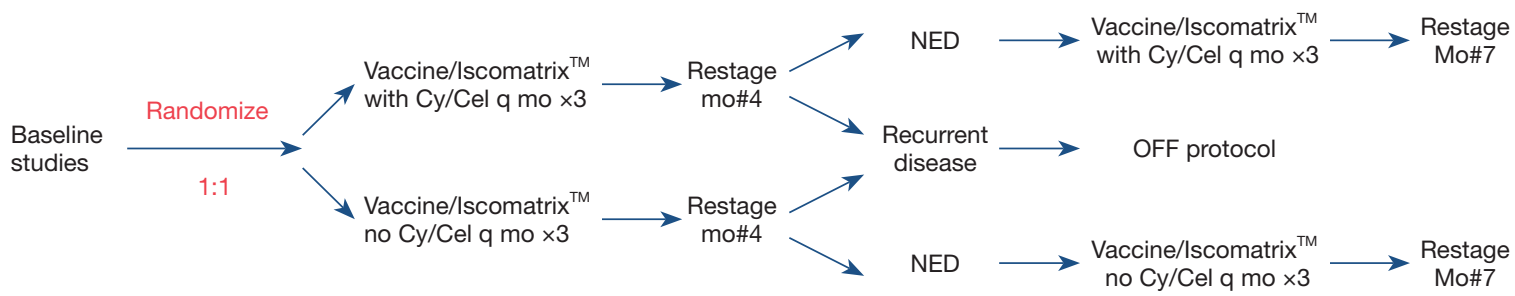

Figure 1 Protocol treatment schema.

care treatment for histologically or cytologically proven primary thoracic malignancies (localized or metastastatic) or neoplasms metastatic to the chest were potentially eligible for study. Patients had to enroll within 52 weeks following definitive therapy for their malignancies, and were required to commence protocol treatment within 4 weeks of eligibility screening and enrollment. Additional eligibility criteria included resolution of all toxicities from prior therapies to $\leq$ Grade 2, adequate bone marrow reserve, as well as hepatic and renal function, no unstable cardiovascular disease, and no decompensated pulmonary disease. Patients requiring chronic systemic steroids at supraphysiologic doses were ineligible, as were patients for whom anticoagulants could not be held for up to 24 hours. Patients with active brain metastases, as well as patients with active infections, and pregnant females were ineligible.

\section{Vaccine production}

The H1299 lung cancer line was obtained from the American Type Culture Collection (ATCC, Manassas, VA). A master bank was established at NCI, Frederick after extensive studies confirmed that the cells were free of mycoplasma, as well as bacterial and viral contaminants. For vaccine production, H1299 cells were seeded into T-1000 flasks and cultured in RPMI media containing 10\% fetal bovine serum (FBS) and Pen/Strep until confluent (approximately 7 days) without exchange of media. Cells were harvested by trypsinization, and extensively washed in HBSS with centrifugation. Cell pellets were then lysed using a freeze-thaw method and centrifuged at 1,000 rpm for 10 minutes to remove debris. Supernatants were diluted to protein concentrations of $10 \mathrm{mg} / \mathrm{mL}$ in normal saline, and then aliquoted into cryotubes $(1.2 \mathrm{~mL} /$ tube $)$ and frozen at $-80{ }^{\circ} \mathrm{C}$. The vaccines were produced in several large batches; approximately $10 \%$ of vials from each preparation were transferred to the NIH Clinical Microbiology Lab to confirm sterility and absence of endotoxin. Immunoblot techniques using primary and secondary antibodies listed in Table S1 were used to confirm levels of NY-ESO-1, MAGE-A1, and MAGE-A3 prior to certification and release of the final product.

\section{Protocol design}

This Phase 2.5 first-in-human trial was conducted at the Center for Cancer Research, NCI, Bethesda, MD. During registration by $\mathrm{CRO}$, patients were randomized 1:1 via non-sequential computer algorithm to receive lysate $(10 \mathrm{mg}$ protein/vaccine) with Iscomatrix ${ }^{\mathrm{TM}}$ adjuvant via deep intradermal injection q 28 days $\times 6$ with or without daily oral metronomic cyclophosphamide and celecoxib (treatment schema depicted in Figure 1). No oral placebo was used; as such, neither patients nor staff were blinded to the treatment regimens. All vaccinations were administered in the same extremity for each patient. Following each vaccination, patients were monitored for approximately one hour for systemic hypersensitivity reactions. A diary was used to document compliance with protocol medications and record systemic toxicities. Each vaccination cycle was 28 days. Cyclophosphamide was administered at a dose of $50 \mathrm{mg} \mathrm{PO}$ BID for 7 days prior to the first vaccination (Day 1, cycle 1), and then on Days 8 through 14, and 22 through 28 of each treatment cycle. Celecoxib was administered at a dose of $400 \mathrm{mg}$ PO BID for 7 days prior to the first vaccination, and then on Days 1 through 28 of each treatment cycle. If vaccinations were delayed, patients continued to take cyclophosphamide every other week and celecoxib daily. Contrast enhanced CT scans of the chest, abdomen and pelvis, torso PET/CT scans, and brain MR scans were obtained at baseline, and one month following the third and sixth vaccinations. Patients who experienced dose limiting toxicities possibly, probably, or definitely attributable to the lysate vaccines received no further vaccines; dose reductions of cyclophosphamide and celecoxib were permissible. Patients who experienced recurrent or new malignancies 
were removed from study and followed until death. Patients who had no detectable immune responses to the vaccine regimen per criteria defined below (non-responders) were also removed from study and continued surveillance in our clinic. The first and last patients were enrolled on 9/3/2014, and $5 / 6 / 15$, respectively, and date of last follow-up was May $15,2020$.

\section{Toxicity assessment, protocol stopping rules, and off-study criteria}

CTCAE version 4.0 was used to grade toxicities. Dose limiting toxicity (DLT) was defined as any Grade 2 or greater autoimmune or hypersensitivity reaction attributable to the vaccine, or Grade 3 or greater toxicity at least possibly related to the vaccine except for Grade 3 toxicities such as local injection site reactions, fatigue, fever, or local adenopathy that resolved to Grade 2 or less within 2 weeks following any vaccination. Protocol stopping rules included any autoimmune DLT at least possibly related to the vaccine that occurred in 3 of the first 10 patients who received study treatment. Criteria for removal from protocol therapy included completion of protocol therapy, vaccine-related DLT, voluntary withdrawal, disease progression, need for systemic steroids, or lack of immune response to vaccine.

\section{Study endpoints and statistical analysis}

The primary endpoint was serologic response to a panel of purified CTA one month after the six vaccination, as evidenced by the appearance of serologic reactivity in previously sero-negative patients, IgM-IgG class switch, or a two-fold or greater increase in titer of IgGs recognizing CT-X antigens. Secondary endpoints included examining if oral metronomic cyclophosphamide (CP) and celecoxib therapy increased frequency or magnitude of immune responses to the lysate vaccines. Exploratory endpoints included evaluation of immune subsets in peripheral blood before and after vaccinations with Wilcoxon matched-pairs signed rank test being used to generate p values (unadjusted for multiple variables); the Mann-Whitney $U$ test was used to compare the two treatment groups.

Following the principles of a phase 2.5 design, the trial was intended to randomize 28 evaluable patients per arm to either receive vaccine alone or vaccine plus chemotherapy in order to have $80 \%$ power to determine if the frequency of immune responses on the combination arm exceeded that of the vaccine alone arm, if the expected frequencies of immune responses on the two arms were $20 \%$ and $50 \%$ using a one-sided 0.10 alpha level Fisher's exact test. The accrual ceiling was set at 60 patients. Despite rapid enrollment, the trial was voluntarily suspended after accrual of only 21 patients due to a sudden permanent loss of access to Iscomatrix ${ }^{\mathrm{TM}}$ or similar adjuvant, and is formally closed as of $5 / 6 / 2021$.

Survival data from 21 patients were analyzed using Kaplan-Meier curves. The difference between the two groups was determined using a log-rank test. Median overall survival with confidence intervals and $\mathrm{p}$ values are reported.

\section{Evaluation of serologic responses to CTA and carbohydrate antigens}

Serologic responses to purified CTA were assessed in a blinded manner by ELISA as previously described (17) using pre- and post-treatment sera at 1:100, 1:400, 1:1,600, and 1:6,400 dilutions. Antibody titers were extrapolated based on a cutoff determined from a pool of healthy donor sera and were considered significant if $>100$.

\section{Analysis of peripheral immune subsets}

Cryopreserved, viable PBMCs were thawed and stained for immune subset analysis as previously reported (18). The following immunophenotypic markers were used to define immune subsets: $\mathrm{CD} 4^{+}$T-cells were $\mathrm{CD} 8^{-} \mathrm{CD} 4^{+}$; $\mathrm{CD} 8^{+}{ }^{-}$-cells were $\mathrm{CD} 4^{-} \mathrm{CD} 8^{+}$; Tregs were $\mathrm{CD} 45^{+} \mathrm{CD} 8^{-}$ $\mathrm{CD} 4^{+} \mathrm{CD} 25^{\text {hi }} \mathrm{Foxp}^{++}$; monocytes were $\mathrm{CD} 14^{+}$, classical monocytes were $\mathrm{CD} 14^{++} \mathrm{CD} 16^{-}$; non-classical monocytes were $\mathrm{CD} 14^{+}, \mathrm{CD} 16^{++}$and intermediate monocytes were $\mathrm{CD} 14^{++} \mathrm{CD} 16^{+}$. Antibodies used for these assays are listed in Table S1. Samples were run on a MACSQuant flow cytometer (Miltenyi Biotec, Bergisch Gladbach, DE), and analyzed using FlowJo software version 10.6.1 (FlowJo LLC, Ashland, OR). Wilcoxon matched-pairs signed rank test was used to generate $\mathrm{p}$ values (unadjusted for multiple variables) for analysis of pre/post treatment assays combining all patients eligible for evaluation;

\section{Results}

\section{CT antigen expression in H1299 lysates}

While screening a large panel of lung cancer lines for NYESO-1 and HLA expression, we identified the H1299 


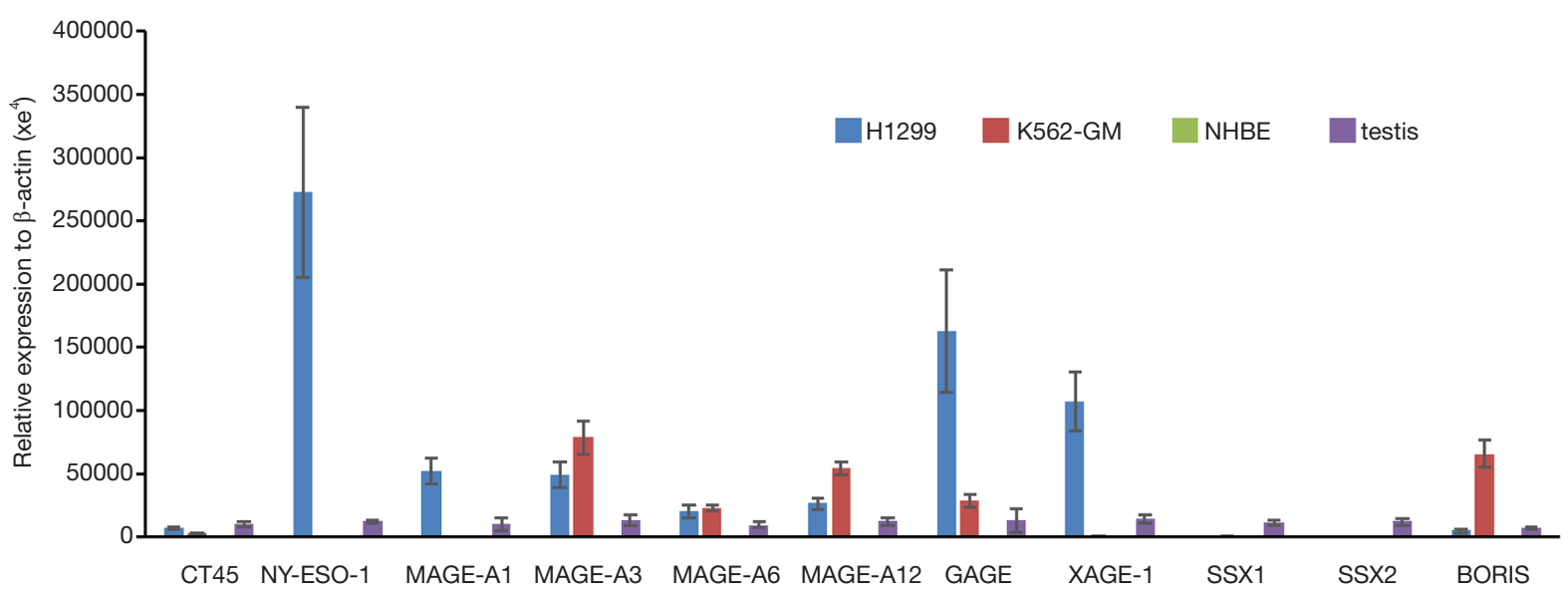

Figure 2 qRT-PCR analysis demonstrating elevated CT-X gene expression in H1299 cells.

large cell lung cancer line as having high level expression of NY-ESO-1 and other $C T$ - $X$ genes typically upregulated in epithelial malignancies (7). Representative qRT-PCR values for a panel of CT genes in H1299 cells are depicted in Figure 2. Notably, H1299 cells express numerous CT-X genes (except for SSX family members that are activated in synovial sarcomas) at levels exceeding those observed in normal testes, and in general at higher levels than K562GM cells that were used for a previous live cell vaccine targeting MAGE-A3 in patients with thoracic malignancies at the NCI (D. Schrump; unpublished). These genes are not expressed in normal respiratory epithelial cells. IHC analysis demonstrated that in contrast to focal CTA expression typically observed in epithelial cancer cells (10), H1299 cells exhibit homogeneous CT-X antigen expression (Figure $3 A$ ) due in part to stable amplification of the X-chromosome (19).

H1299 cells express class I as well as class II MHC antigens (data available upon request); consequently, when used as live, irradiated cell vaccines, these cells could induce allo-reactivity that might limit responses to CT-X antigens. As such, experiments were performed to examine if freezethaw lysate techniques could be used to deplete HLA proteins while preserving CT-X antigen levels. As shown in Figure 3B, total protein yields were not significantly different in freezethaw lysates compared to conventional RIPA buffer lysates; Decitabine treatment did not significantly increase yields, presumably due to endogenous maximal demethylation of the respective CT-X gene promoters. Immunoblot analysis (Figure 3C) demonstrated that freeze-thaw lysis markedly decreased levels of class I MHC proteins while retaining levels of CT-X antigens such as NY-ESO-1, MAGE-A1 and
MAGE-A3 relative to RIPA lysates.

\section{Patient demographics}

Of 25 patients screened, 4 were deemed ineligible due to detection of active disease $(n=3)$ or withdrawal prior to randomization (n=1; Figure 4). 11 patients with primary thoracic malignancies and 10 patients with extra-thoracic neoplasms metastatic to the chest were enrolled in the study (Table 1). The median age of all patients was 51 years (range, 18.6-73.3); of note, median age of patients with primary thoracic malignancies was significantly higher than that of patients with cancers metastatic to the chest (61 vs. 36 years; $\mathrm{P}<0.05)$. Twelve $(57 \%)$ of the patients were male. 8 patients (38\%) had lung cancer; another 8 patients had sarcomas. 8 patients had locally advanced or metastatic disease at diagnosis and were rendered NED by surgery, chemotherapy, CRT, or combined modality treatment, whereas 7 developed metachronous metastases that were resected to render them NED prior to protocol enrollment. Fourteen patients $(67 \%)$ received prior chemotherapy, whereas $12(57 \%)$ received prior XRT. The melanoma patient had received several prior immunotherapy regimens. Three patients had previously participated in a live-irradiated K562-GM vaccine trial in our Branch (DSS; unpublished); none of these patients had exhibited an immunologic response to purified MAGE-A3 (the primary endpoint of that trial).

\section{Clinical responses in vaccinated patients}

All patients exhibited local inflammatory responses 
A

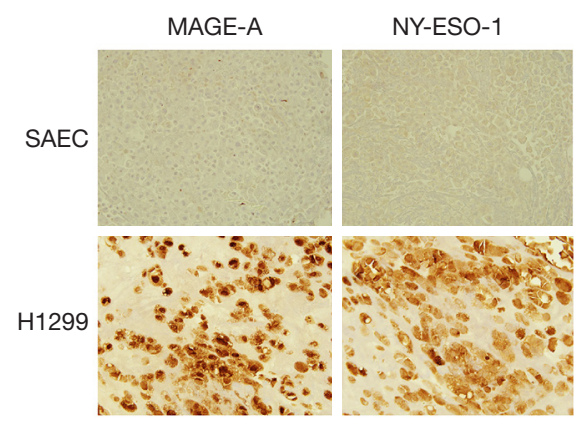

C

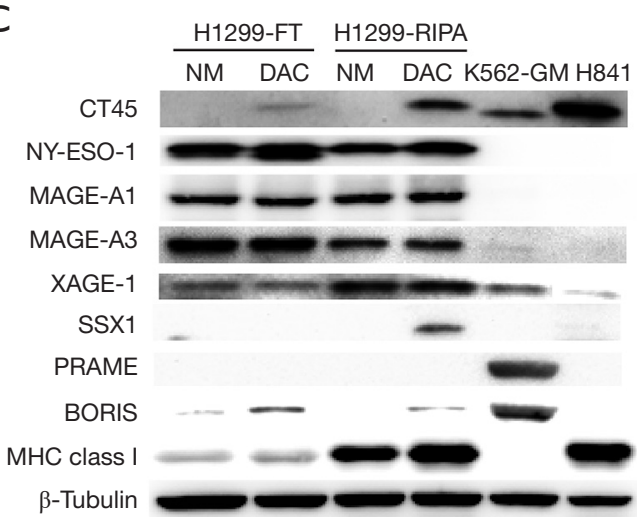

B

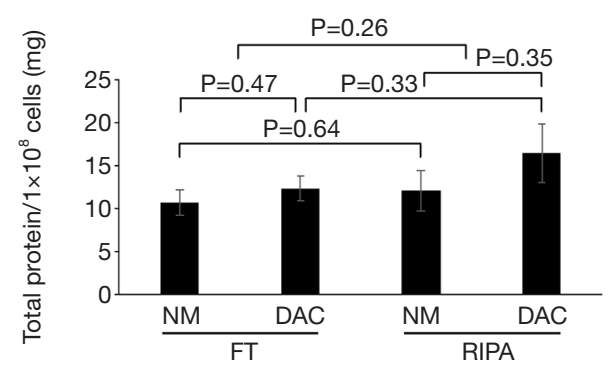

Figure 3 Cancer-testis antigen expression in cultured H1299 lung cancer cells and cell lysates. (A) IHC analysis $(\times 20)$ showing increased CT-X antigen expression in H1299 cells in a homogeneous manner compared to normal respiratory epithelial cells (SAEC). (B) Total protein yield in lysates from $1 \times 10^{8} \mathrm{H} 1299$ cells by freeze-thaw (FT) vs. RIPA buffer. (C) Immunoblot analysis demonstrating that freezethaw lysis decreased levels of MHC class I proteins compared to RIPA lysates while retaining levels of CT-X antigens. Levels of NY-ESO-1 ans MAGE proteins are much higher in H1299 lysates relative to HLA-deficient K562-GM cells or H841 lung cancer cells.

(Figure 5A) and flu-like symptoms lasting 72-96 hours following vaccinations. There were no dose limiting treatment related toxicities. 14 patients (67\%) including 9 with primary thoracic malignancies completed all six vaccinations and were evaluable for treatment response (Figure 4). Four patients (one with melanoma, 3 with sarcomas) were removed from study early due to disease recurrence. An additional sarcoma patient voluntarily withdrew from study due to college commitments. One lung cancer patient was removed from study early due to second primary NSCLC, and one NSCLC patient was removed from study due to difficulty assessing disease status in an irradiated mediastinum and was deemed to have progressive disease.

\section{Molecular responses in vaccinated patients}

Eight of 14 patients (57\%) exhibited unequivocal serologic responses to NY-ESO-1; 6 of these 8 responders (75\%) had primary thoracic malignancies. Six of 9 patients (67\%) with primary thoracic malignancies compared to two of five patients $(40 \%)$ with pulmonary metastases who completed the vaccine regimen exhibited unequivocal serologic responses to NY-ESO-1. One patient developed antibodies to GAGE7; several patients exhibited reactivity to XAGE and MAGE-C2. Results of the analysis are summarized in Table 2, and Figure 5B. Vaccine therapy reduced the percent of Tregs $(\mathrm{P}=0.0068)$, and decreased intensities of $\mathrm{PD}-1$ expression on Tregs $(\mathrm{P}=0.0027)$, $\mathrm{PD}$ $\mathrm{L} 1$ expression on monocytes $(\mathrm{P}=0.0089)$, $\mathrm{PD}-\mathrm{L} 1$ expression on classical monocytes $(\mathrm{P}=0.016)$, and $\mathrm{PD}-\mathrm{L} 1$ expression on intermediate monocytes $(\mathrm{P}=0.0031)$ (Figure 6). Metronomic cyclophosphamide/celecoxib did not increase immune responses or enhance vaccine-induced alterations in peripheral immune subsets (results pertaining to peripheral blood Tregs are depicted in Figure S1). Additional analysis revealed no associations between metrics of immunologic responses and survival, possibly due to the limited number 


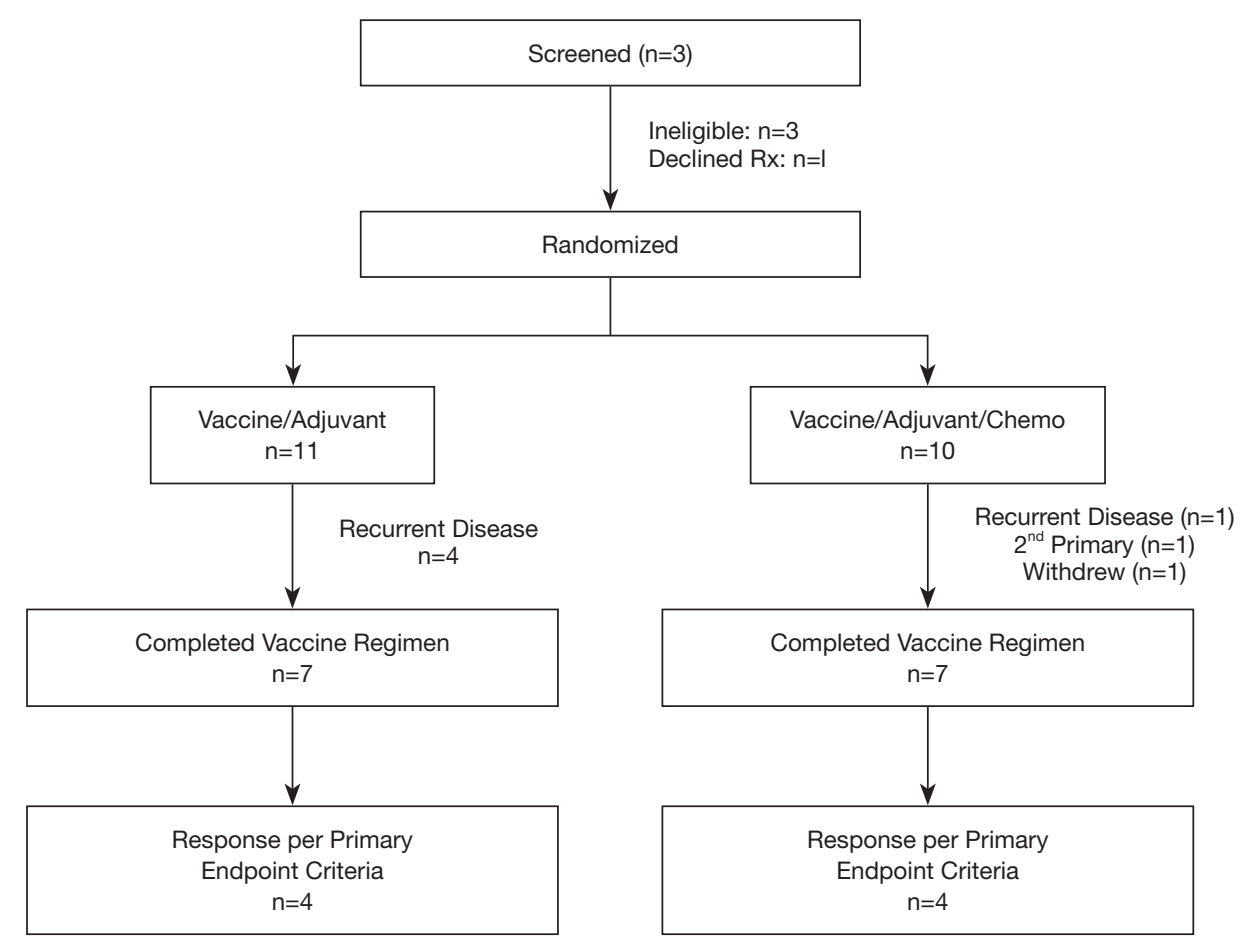

Figure 4 Trial participant flow.

of patients on this trial.

\section{Clinical outcomes}

Although not a stated objective in the protocol, outcome data are available for all patients enrolled on the trial. With a median follow-up of 62.1 months (range, 59-68.7), 12 patients (2 SCLC, 3 NSCLC, 2 EsC, 4 sarcoma, 1 RCC) are alive and NED, and 2 patients (both with NSCLC) are alive with new, but controlled malignancies (one DLBCL; one second primary NSCLC). 11 of the 14 surviving patients $(79 \%)$ received all six vaccinations. In contrast, of the 7 patients ( 4 sarcoma, 1 melanoma, 1 TET, 1 NSCLC) who succumbed to their malignancies, only 3 (42\%) received all six vaccinations.

Ten patients randomized to vaccine with metronomic cyclophosphamide/celecoxib. Seven of these patients received all six vaccinations. Two of these 7 patients were removed from study one month following the $6^{\text {th }}$ vaccination due to disease recurrence; these two individuals and one more of the seven patients (43\%) subsequently died of disease. Eleven patients randomized to vaccine alone. Seven of these patients completed all six vaccinations; none of these patients ( $0 \%$ ) have died (Figure 7). There were no obvious associations between histology or stage of tumors and outcomes in these patients.

\section{Discussion}

The highly restricted expression, potential oncogenic activities, and immunogenicity of CTA makes them attractive targets for cancer immunotherapy $(4,5)$. However, limited information is available regarding the potential efficacy of vaccines targeting these proteins in patients with thoracic malignancies. In general, immunologic responses to these vaccines are more evident in patients with no evidence of disease or minimal disease burden relative to patients with extensive, bulky disease (20); this was our rationale for evaluating the H1299 lysate vaccine in the adjuvant setting. Atanackovic et al. (21) observed that a vaccine containing recombinant whole protein MAGE-A3 with a proprietary immunostimulant (AS15 or AS02B) induced $\mathrm{CD}^{+}$and $\mathrm{CD}^{+}$immune responses in lung cancer patients. Furthermore, a Phase II trial suggested that this vaccine might prolong survival in certain subsets of lung cancer patients undergoing definitive resection (22). Immunologic responses in lung cancer patients coincided with an 84 gene signature identified in tumor tissues of 
Table 1 Patient demographics

\begin{tabular}{|c|c|c|c|c|c|c|c|c|}
\hline Patient \# & Gender & Age, years & Histology & Stage & Prior surg & Prior chemo & Prior XRT & Prior immuno \\
\hline 1 & $\mathrm{~F}$ & 33.9 & TET & IVA & Y & Y & Y & $\mathrm{N}$ \\
\hline 2 & $M$ & 43.2 & SCLC & LS & $\mathrm{N}$ & Y & Y & $\mathrm{N}$ \\
\hline 3 & $M$ & 55.1 & NSCLC & $\mathrm{IA}$ & Y & $N$ & $\mathrm{~N}$ & $\mathrm{~N}$ \\
\hline 4 & $M$ & 45.9 & SAR & IV & Y & Y & Y & $\mathrm{N}$ \\
\hline 5 & $M$ & 23.9 & SAR & IV & Y & Y & Y & $\mathrm{N}$ \\
\hline 6 & $M$ & 40.6 & ACC & III & Y & $N$ & $N$ & $\mathrm{~N}$ \\
\hline 7 & M & 39.5 & SAR & IV & Y & $\mathrm{Y}$ & Y & $\mathrm{N}$ \\
\hline 8 & $\mathrm{~F}$ & 68.3 & NSCLC & IB & Y & $N$ & $\mathrm{~N}$ & $\mathrm{~N}$ \\
\hline 9 & $M$ & 35 & SAR & IV & Y & $\mathrm{N}$ & $Y$ & $\mathrm{~N}$ \\
\hline 10 & $\mathrm{~F}$ & 59.5 & NSCLC & IA & Y & $N$ & $\mathrm{~N}$ & $\mathrm{~N}$ \\
\hline 11 & $\mathrm{~F}$ & 21.7 & SAR & IV & Y & Y & Y & $\mathrm{N}$ \\
\hline 12 & $M$ & 63.8 & NSCLC & $\| A$ & Y & Y & $\mathrm{N}$ & $\mathrm{N}$ \\
\hline 13 & $\mathrm{~F}$ & 65.1 & ESC & IV & $\mathrm{N}$ & Y & $\mathrm{N}$ & $\mathrm{N}$ \\
\hline 14 & $M$ & 18.6 & SAR & IV & Y & Y & $\mathrm{N}$ & $\mathrm{N}$ \\
\hline 15 & $\mathrm{~F}$ & 58.3 & SAR & IV & Y & Y & Y & $\mathrm{N}$ \\
\hline 16 & $M$ & 51.2 & ESC & IIIA & Y & Y & $Y$ & $\mathrm{~N}$ \\
\hline 17 & $\mathrm{~F}$ & 63.5 & NSCLC & IA & Y & $N$ & $\mathrm{~N}$ & $\mathrm{~N}$ \\
\hline 18 & $M$ & 65.2 & NSCLC & IIIA & Y & Y & Y & $\mathrm{N}$ \\
\hline 19 & $\mathrm{~F}$ & 21.8 & SAR & IV & Y & $\mathrm{Y}$ & $\mathrm{Y}$ & $\mathrm{N}$ \\
\hline 20 & $M$ & 73.3 & MEL & IV & Y & Y & $\mathrm{N}$ & Y \\
\hline 21 & $\mathrm{~F}$ & 51.9 & SCLC & LS & $\mathrm{N}$ & Y & $\mathrm{Y}$ & $\mathrm{N}$ \\
\hline
\end{tabular}

TET, thymic epithelial tumor; SCLC, small cell lung cancer; NSCLC, non-small cell lung cancer; SAR, sarcoma; ACC, adrenocortical carcinoma; ESC, esophageal cancer; MEL, melanoma; LS, limited stage.

melanoma patients responding to MAGE-A3 vaccines (22); the majority of genes predictive of response to vaccines were immune-related. In another trial, NY-ESO-1 DNA vaccines mediated $\mathrm{CD}^{+} \mathrm{T}$ cell responses in 5 of 5 patients with advanced stage NSCLC, three of whom developed $\mathrm{CD}^{+} \mathrm{T}$ cell responses to purified NY-ESO-1 peptides (23). Two lung cancer patients with measurable disease at the start of vaccination exhibited stable disease for 16 and 23 months; two additional lung cancer patients and one esophageal cancer patient with Stage IV disease who were NED at the time of protocol entry remained free of disease.

One potential strategy to enhance immune responses to cancers exhibiting low-level, heterogeneous expression of CTA and immune-related genes (24-26) involves vaccination with autologous cells genetically engineered to express high-levels of these potential targets. Fontana et al. (27) observed immunologic responses to MAGE-A3 in 3 of $10(30 \%)$ melanoma patients vaccinated with autologous PBL genetically-modified to express this CT-X antigen. Russo et al. (28) observed immunologic responses to MAGE-A3 in 6 of 23 melanoma patients receiving autologous lymphocytes genetically engineered to express this antigen. Of 19 patients with measurable disease, one had an objective response, and four patients exhibited durable stabilization of disease.

An alternative strategy is to use allogeneic cells to immunize cancer patients against multiple CTA that potentially can be up-regulated specifically in tumor cells by aforementioned systemic gene induction regimens (7). A recent report by Safavi et al. (29) demonstrating that 
A
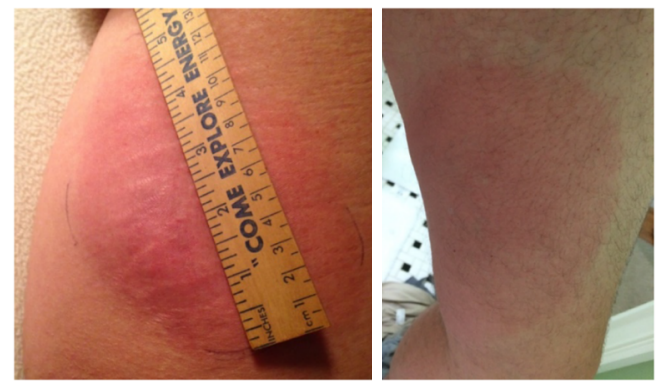

B

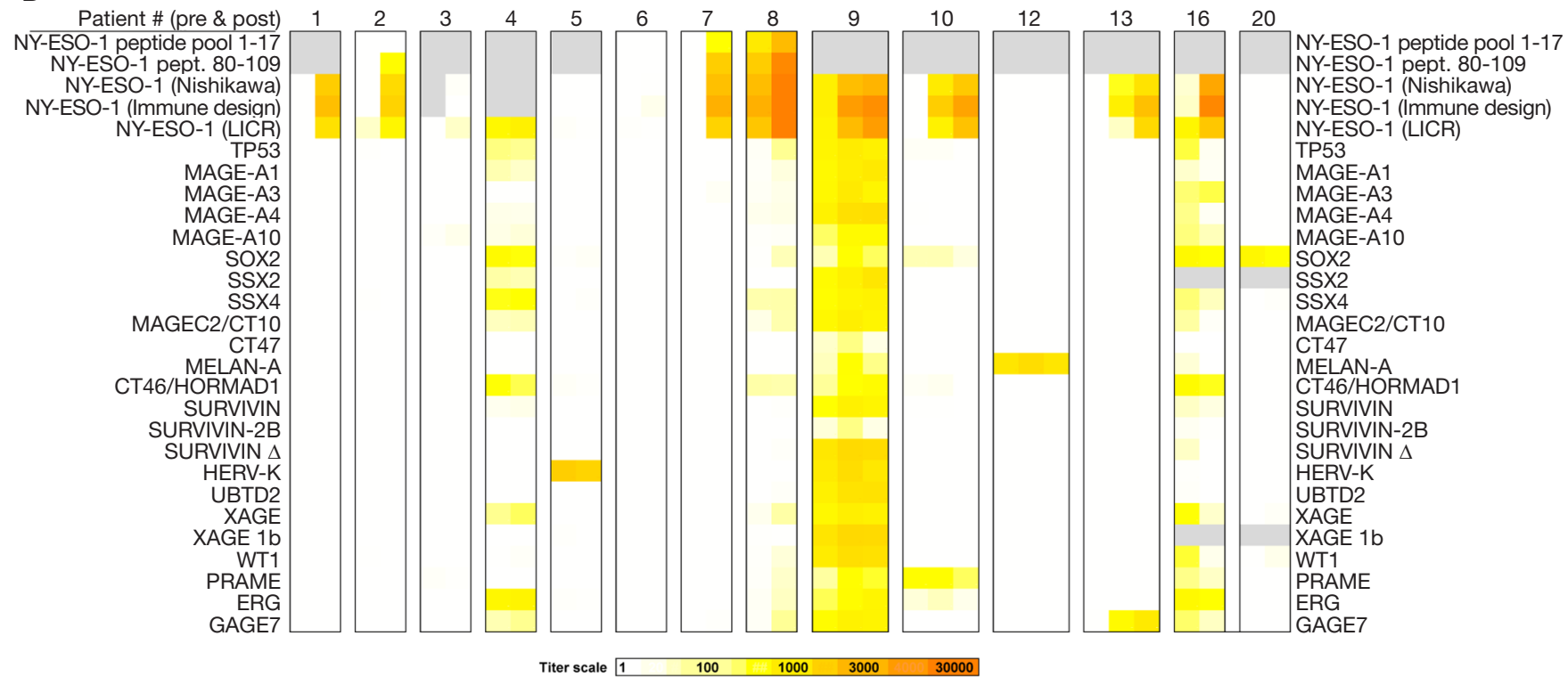

Figure 5 Local and systemic effects of H1299 lysate vaccine with Iscomatrix ${ }^{\mathrm{TM}}$ adjuvant in cancer patients. (A) Representative photos from two patients demonstrating local inflammatory responses at the injection sites approximately $48 \mathrm{~h}$ following vaccinations. (B) Analysis of serologic responses to a panel of potential tumor associated antigens including CT-X and non-CT-X antigens in pre- and post-vaccination patient sera.

a testicular germ cell vaccine administered with BCG significantly reduces growth and lethality of 4T1 breast cancer cells in a murine cancer model supports our approach in humans. In the present study we utilized the H1299 lung cancer line to immunize thoracic oncology patients against potentially relevant CTA focusing primarily on responses to CT-X antigens. We observed a relatively high percentage of patients with objective responses to NY-ESO-1. Despite these encouraging results, we did not observe significant responses to MAGE family members which are among the most commonly expressed CT-X antigens in thoracic malignancies $(30,31)$. This may be due to amounts and relative immunogenicities of CT-X antigens in the vaccine lysates, as well as the stringency regarding what was defined as antibody responses in our trial. Pujol et al. (32) observed variable antibody responses in all lung cancer patients who received MAGE-A3 immunotherapeutic (300 $\mu \mathrm{g}$ purified protein with proprietary adjuvant) $\mathrm{q} 3$ weeks $\times 8$ weeks. The lack of toxicities associated with the immunization regimen in our trial suggests that the protein content per vaccination can be safely increased to possibly broaden immune responses to CT-X antigens.

$\mathrm{CD} 4{ }^{+} \mathrm{CD} 25^{+} \mathrm{T}$ cells expressing the forkhead box $\mathrm{P} 3$ (Fox P3) transcription factor mediate immune tolerance in cancer patients $(33,34)$. Chronic inflammation within tumors facilitates conversion of FoxP3 ${ }^{+} \mathrm{T}_{\mathrm{H}}$ cells to a suppressive phenotype (35). Absolute numbers and percentages of Tregs within the tumor microenvironment and peripheral blood 
Table 2 Summary of treatment responses

\begin{tabular}{lccccccccc}
\hline Patient \# & Histology & ECOG & Cohort & \# Vaccines & Toxicity & Response & Off study reason & Status & OS, months \\
\hline 1 & TET & 0 & VC & 6 & N & Y & PD & DOD & 56.6 \\
2 & SCLC & 0 & V & 6 & N & Y & on study & NED & 67.7 \\
3 & NSCLC & 0 & V & 6 & N & N & NR & NED & 64.7 \\
4 & SAR & 0 & V & 6 & N & INC & NR & NED & 64.6 \\
5 & SAR & 0 & VC & 6 & N & N & NR & DOD & 21.6 \\
6 & ACC & 0 & VC & 6 & N & N & NR & NED & 65.8 \\
7 & SAR & 0 & VC & 6 & N & Y & on study & NED & 65.8 \\
8 & NSCLC & 0 & VC & 6 & N & Y & PD & DOD & 25.4 \\
9 & SAR & 0 & V & 6 & N & Y & on study & NED & 64.8 \\
10 & NSCLC & 0 & V & 6 & N & Y & DLBCL & DLBCL & 64.8 \\
11 & SAR & 1 & V & 3 & N & N/A & PD & DOD & 27.2 \\
12 & NSCLC & 0 & VC & 6 & N & N & NR & NED & 64.6 \\
13 & ESC & 0 & V & 6 & N & Y & On study & NED & 64.6 \\
14 & SAR & 0 & V & 3 & N & N/A & PD & DOD & 22.2 \\
15 & SAR & 0 & VC & 3 & N & N/A & PD & DOD & 20.9 \\
16 & ESC & 0 & VC & 6 & N & Y & On study & NED & 62.1 \\
17 & NSCLC & 0 & VC & 3 & N & N/A & NSCLC* & NED & 62.1 \\
18 & NSCLC & 0 & V & 3 & N & N/A & PD & NED & 61.2 \\
19 & SAR & 0 & VC & 2 & N & N/A & Withdrew & NED & 59.8 \\
21 & MEL & 1 & V & 2 & N & N/A & PD & DOD & 7.4 \\
\hline & SCLC & 0 & V & 6 & N & N & NR & NED & 60.3 \\
\hline
\end{tabular}

*, 2nd primary. TET, thymic epithelial tumor; SCLC, small cell lung cancer; NSCLC, non-small cell lung cancer; SAR, sarcoma; ACC, adrenocortical carcinoma; ESC, esophageal cancer; MEL, melanoma. NR, No response.

of cancer patients correlate with advanced stage of disease and poor survival in these individuals $(34,36)$.

Several studies have suggested that the capacity of cancer vaccines to induce effective antitumor immunity may be abrogated by simultaneous expansion of vaccineinduced immunosuppressive Tregs $(37,38)$. Of particular relevance regarding induction of immunity to CT-X antigens are observations that Tregs attenuate immune responses to NY-ESO-1 vaccines in patients with melanoma as well as prostate, lung, and esophageal carcinomas $(20,23)$. In the current study we utilized oral metronomic cyclophosphamide and celecoxib as a strategy to target activity of Tregs $(39,40)$ yet did not observe any significant impact of the regimen with respect to frequency or magnitude of serologic responses to the lysate vaccines, possibly due to the limited number of patients on the trial. Interestingly, the vaccine regimen did modulate peripheral immune subsets in a manner which theoretically could enhance antitumor immunity. Rather than expand Tregs, the vaccine, with or without cyclophosphamide and celecoxib resulted in a significant decrease in peripheral Tregs and a decrease in the expression of PD-1 on Tregs, which is consistent with a decrease in Treg immunosuppressive activity (41). This was accompanied by a potentially favorable modulation of innate immunity, as shown by a decrease in PD-L1 expression on total monocytes and monocyte subsets (42). Whether this was related to the lysate vaccine, the potent adjuvant, or both is unknown at present.

Limitations of the present study include early termination 
A

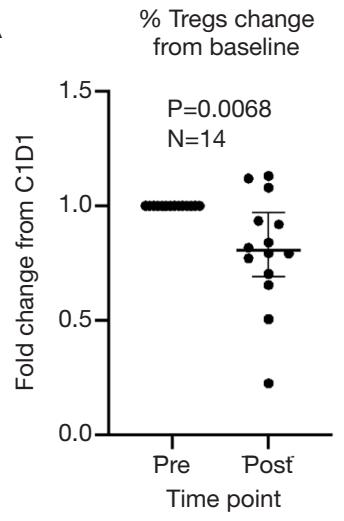

B

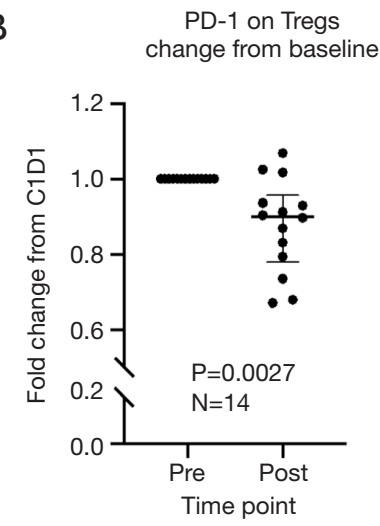

C

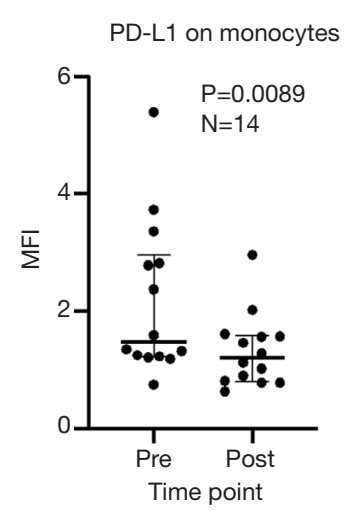

$\mathrm{D}$

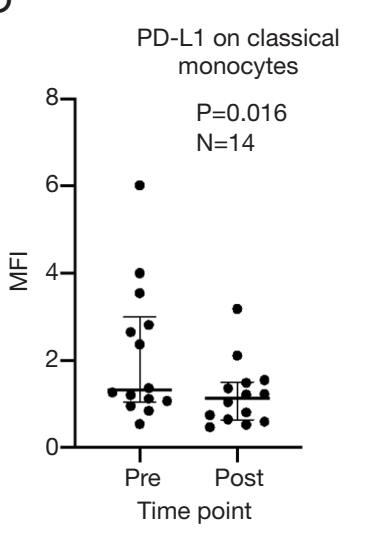

$\mathrm{E}$

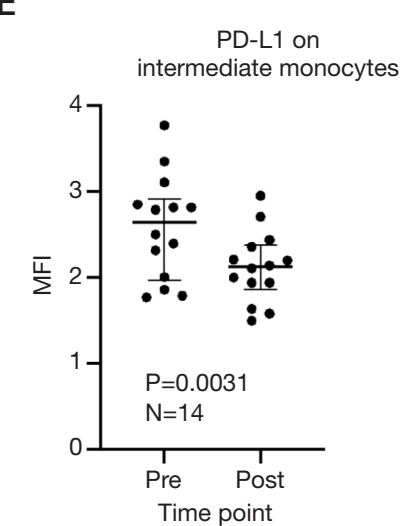

Figure 6 Flow cytometry analysis of peripheral immune subsets in cancer patients before and after H1299 lysate and iscomatrix ${ }^{\mathrm{TM}}$ treatment regimen. (A) Changes in percent Tregs among $\mathrm{CD}^{+} \mathrm{T}$ cells (B) Changes in intensities of PD-1 expression [median fluorescence intensity (MFI)] on Tregs. (C,D,E) Changes in intensities of PD-L1 expression (MFI) on total monocytes, classical monocytes, and intermediate monocytes, respectively. Wilcoxon matched-pairs signed rank test was used to generate $\mathrm{P}$ values (unadjusted for multiple variables).

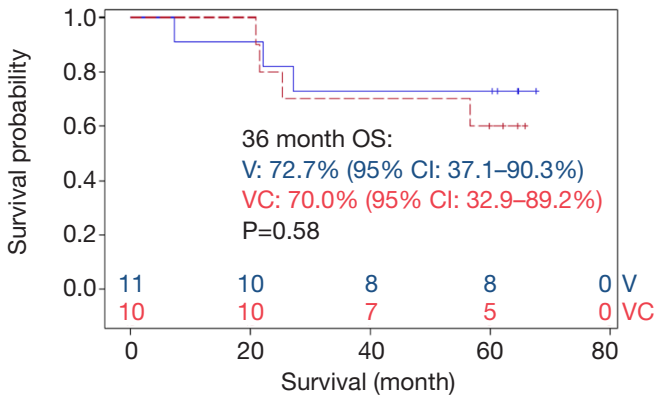

Figure 7 OS of patients randomized either to vaccine with adjuvant or vaccine with adjuvant and metronomic cyclophosphamide/ celecoxib.

due to sudden, permanent loss of access to Iscomatrix and inability to obtain a similar type of adjuvant to continue the trial. Production of the lysate was halted for several years due to restrictions imposed by the NIH following the Red Team Report (https://www.sciencemag.org/ news/2016/06/nih-uproar-over-report-slamming-clinicalcenter-leadership-shakeup), which among other issues was highly critical of methods of production of cell based products for early phase clinical trials; extensive renovations and additional oversight of all production facilities imposed by the NIH resulted in prolonged voluntary suspension of the trial; this issue has now been resolved. Additionally, the trial included a heterogeneous group of patients (one cohort of 11 patients with primary thoracic malignancies of whom $9(82 \%)$ completed the vaccine regimen, and another cohort of 10 patients with pulmonary metastases from extra-thoracic primaries, of whom only 5 (50\%) completed the investigational treatment). These findings suggest that the vaccine regimen is not feasible in these latter patients, possibly due to histology and burden of 
subclinical metastatic disease, as well as underlying immune status. The lack of primary tumor tissues from most of the patients prevented analysis of baseline CTA expression and correlation with serum antibody titers. Given the prolonged voluntary suspension related to adjuvant availability and new lysate production, this trial has been closed. A new trial will be initiated in the near future utilizing a higher lysate dose and Montanide ${ }^{\circledR}$ ISA 51 VG adjuvant with or without the IL-15 superagonist, $\mathrm{N}-803$ in an attempt to induce immunity to more CT-X antigens in lung cancer patients from whom baseline tumor tissues are available for assessment of antigen expression and evaluation of serologic as well as cell mediated immune responses to autologous tumor cell lysates and lung cancer stem cells.

\section{Conclusions}

H1299 cell lysate vaccines can induce immunity to potentially relevant CTA in thoracic malignancies, and modulate peripheral immune subsets in a manner that may enhance anti-tumor immune responses. Lack of systemic toxicities suggests that the vaccine dose can be safely increased. Despite premature suspension of the trial, our findings support further analysis of the lysate vaccine in combination with either cytokines or immune checkpoint inhibitors as a strategy to target dormant cancer stem cells in thoracic malignancies.

\section{Acknowledgments}

The authors thank Ms. Nina Solarz, Randy Glantz, Leah Glantz, and Ariana Glantz for their continued support of our translational research efforts, Seth M. Steinberg for assistance with data analysis, and Ms. Jan Pappas for administrative support. The authors also wish to express our gratitude to all of the patients who enrolled on this vaccine trial.

Funding: The preclinical studies and protocol were supported by NIH intramural funds (ZIA SC 010093; DSS), and the Stephen J. Solarz Memorial Fund at the Foundation of the National Institutes of Health (FNIH).

\section{Footnote}

Reporting Checklist: The authors have completed the CONSORT reporting checklist. Available at https://dx.doi. org/10.21037/tlcr-21-1

Trial Protocol: Available at https://dx.doi.org/10.21037/ tlcr-21-1

Data Sharing Statement: Available at https://dx.doi. org/10.21037/tlcr-21-1

Conflicts of Interest: All authors have completed the ICMJE uniform disclosure form (available at https:// dx.doi.org/10.21037/tlcr-21-1). Dr. SG is supported by grants U24 CA224319 and U01 DK124165. He reports past consultancy and/or advisory roles for Merck, Neon Therapeutics and OncoMed, and research funding from Bristol-Myers Squibb, Genentech, Immune Design, Agenus, Janssen R\&D, Pfizer, Takeda, and Regeneron, unrelated to current work. JBT receives research funding from Syndax, EpicentRx and AstraZeneca. CDH serves as an unpaid editorial board member of Translational Lung Cancer Research from Jul 2019 to Jul 2021. The authors have no other conflicts of interest to declare.

Ethical Statement: The authors are accountable for all aspects of the work in ensuring that questions related to the accuracy or integrity of any part of the work are appropriately investigated and resolved. The trial was conducted in accordance with the Declaration of Helsinki (as revised in 2013) and the guidelines for Good Clinical Practice. The trial (NCT02054104) was approved by NCIIRB and FDA (IND 15812), and written informed consent was obtained from all individual participants.

Open Access Statement: This is an Open Access article distributed in accordance with the Creative Commons Attribution-NonCommercial-NoDerivs 4.0 International License (CC BY-NC-ND 4.0), which permits the noncommercial replication and distribution of the article with the strict proviso that no changes or edits are made and the original work is properly cited (including links to both the formal publication through the relevant DOI and the license). See: https://creativecommons.org/licenses/by-nc-nd/4.0/.

\section{References}

1. Burnet FM. The concept of immunological surveillance. Prog Exp Tumor Res 1970;13:1-27.

2. Dunn GP, Old LJ, Schreiber RD. The immunobiology of cancer immunosurveillance and immunoediting. Immunity 2004;21:137-48.

3. Simpson AJ, Caballero OL, Jungbluth A, et al. Cancer/ testis antigens, gametogenesis and cancer. Nat Rev Cancer 
2005;5:615-25.

4. Salmaninejad A, Zamani MR, Pourvahedi M, et al. Cancer/ Testis Antigens: Expression, Regulation, Tumor Invasion, and Use in Immunotherapy of Cancers. Immunol Invest 2016;45:619-40.

5. Gibbs ZA, Whitehurst AW. Emerging Contributions of Cancer/Testis Antigens to Neoplastic Behaviors. Trends Cancer 2018;4:701-12.

6. Dashti S, Taherian-Esfahani Z. Cellular immune responses against cancer-germline genes in cancers. Hum Antibodies 2020;28:57-64.

7. Schrump DS. Targeting epigenetic mediators of gene expression in thoracic malignancies. Biochim Biophys Acta 2012;1819:836-45.

8. Weiser TS, Guo ZS, Ohnmacht GA, et al. Sequential 5-Aza-2 deoxycytidine-depsipeptide FR901228 treatment induces apoptosis preferentially in cancer cells and facilitates their recognition by cytolytic $\mathrm{T}$ lymphocytes specific for NY-ESO-1. J Immunother 2001;24:151-61.

9. Wargo JA, Robbins PF, Li Y, et al. Recognition of NYESO-1+ tumor cells by engineered lymphocytes is enhanced by improved vector design and epigenetic modulation of tumor antigen expression. Cancer Immunol Immunother 2009;58:383-94.

10. Rao M, Chinnasamy N, Hong JA, et al. Inhibition of histone lysine methylation enhances cancer-testis antigen expression in lung cancer cells: implications for adoptive immunotherapy of cancer. Cancer Res 2011;71:4192-204.

11. Schrump DS, Fischette MR, Nguyen DM, et al. Phase I study of decitabine-mediated gene expression in patients with cancers involving the lungs, esophagus, or pleura. Clin Cancer Res 2006;12:5777-85.

12. Schrump DS, Fischette MR, Nguyen DM, et al. Clinical and molecular responses in lung cancer patients receiving Romidepsin. Clin Cancer Res 2008;14:188-98.

13. Guo ZS, Hong JA, Irvine KR, et al. De novo induction of a cancer/testis antigen by 5 -aza-2'-deoxycytidine augments adoptive immunotherapy in a murine tumor model. Cancer Res 2006;66:1105-13.

14. Gordeeva O. Cancer-testis antigens: Unique cancer stem cell biomarkers and targets for cancer therapy. Semin Cancer Biol 2018;53:75-89.

15. Yamada R, Takahashi A, Torigoe T, et al. Preferential expression of cancer/testis genes in cancer stem-like cells: proposal of a novel sub-category, cancer/testis/stem gene. Tissue Antigens 2013;81:428-34.

16. Yin B, Zeng Y, Liu G, et al. MAGE-A3 is highly expressed in a cancer stem cell-like side population of bladder cancer cells. Int J Clin Exp Pathol 2014;7:2934-41.

17. Stockert E, Jager E, Chen YT, et al. A survey of the humoral immune response of cancer patients to a panel of human tumor antigens. J Exp Med 1998;187:1349-54.

18. Thomas A, Rajan A, Szabo E, et al. A phase I/II trial of belinostat in combination with cisplatin, doxorubicin, and cyclophosphamide in thymic epithelial tumors: a clinical and translational study. Clin Cancer Res 2014;20:5392-402.

19. Shukla V, Rao M, Zhang H, et al. ASXL3 Is a Novel Pluripotency Factor in Human Respiratory Epithelial Cells and a Potential Therapeutic Target in Small Cell Lung Cancer. Cancer Res 2017;77:6267-81.

20. Nicholaou T, Ebert LM, Davis ID, et al. Regulatory T-cell-mediated attenuation of T-cell responses to the NYESO-1 ISCOMATRIX vaccine in patients with advanced malignant melanoma. Clin Cancer Res 2009;15:2166-73.

21. Atanackovic D, Altorki NK, Stockert E, et al. Vaccineinduced CD4+ T cell responses to MAGE-3 protein in lung cancer patients. J Immunol 2004;172:3289-96.

22. Ulloa-Montoya F, Louahed J, Dizier B, et al. Predictive Gene Signature in MAGE-A3 Antigen-Specific Cancer Immunotherapy. J Clin Oncol 2013;31:2388-95.

23. Gnjatic S, Altorki NK, Tang DN, et al. NY-ESO-1 DNA vaccine induces $\mathrm{T}$-cell responses that are suppressed by regulatory T cells. Clin Cancer Res 2009;15:2130-9.

24. Jin S, Cao S, Grigorev A, et al. Establishment of cancer/ testis antigen profiling based on clinicopathological characteristics in resected pathological stage III non-small cell lung cancer. Cancer Manag Res 2018;10:2031-46.

25. Djureinovic D, Dodig-Crnković T, Hellström C, et al. Detection of autoantibodies against cancer-testis antigens in non-small cell lung cancer. Lung Cancer 2018;125:157-63.

26. Ma KY, Schonnesen AA, Brock A, et al. Single-cell RNA sequencing of lung adenocarcinoma reveals heterogeneity of immune response-related genes. JCI Insight 2019;4:e121387.

27. Fontana R, Bregni M, Cipponi A, et al. Peripheral blood lymphocytes genetically modified to express the self/tumor antigen MAGE-A3 induce antitumor immune responses in cancer patients. Blood 2009;113:1651-60.

28. Russo V, Pilla L, Lunghi F, et al. Clinical and immunologic responses in melanoma patients vaccinated with MAGEA3-genetically modified lymphocytes. Int J Cancer 2013;132:2557-66.

29. Safavi A, Kefayat A, Ghahremani F, et al. Immunization using male germ cells and gametes as rich sources of 
cancer/testis antigens for inhibition of 4T1 breast tumors' growth and metastasis in BALB/c mice. Int Immunopharmacol 2019;74:105719.

30. Sang M, Gu L, Yin D, et al. MAGE-A family expression is correlated with poor survival of patients with lung adenocarcinoma: a retrospective clinical study based on tissue microarray. J Clin Pathol 2017;70:533-40.

31. Ishihara M, Kageyama S, Miyahara Y, et al. MAGE-A4, NY-ESO-1 and SAGE mRNA expression rates and coexpression relationships in solid tumours. BMC Cancer 2020;20:606.

32. Pujol JL, Vansteenkiste JF, De Pas TM, et al. Safety and Immunogenicity of MAGE-A3 Cancer Immunotherapeutic with or without Adjuvant Chemotherapy in Patients with Resected Stage IB to III MAGE-A3-Positive Non-SmallCell Lung Cancer. J Thorac Oncol 2015;10:1458-67.

33. Saleh R, Elkord E. FoxP3(+) T regulatory cells in cancer: Prognostic biomarkers and therapeutic targets. Cancer Lett 2020;490:174-85.

34. Hu X, Gu Y, Zhao S, et al. Elevated Circulating CD4(+) CD25(-)Foxp3(+) Regulatory T Cells in Patients with Nonsmall Cell Lung Cancer. Cancer Biother Radiopharm 2019;34:325-33.

35. Xie M, Wei J, Xu J. Inducers, Attractors and Modulators of CD4(+) Treg Cells in Non-Small-Cell Lung Cancer. Front Immunol 2020;11:676.

36. Kochin V, Nishikawa H. Meddling with meddlers: curbing regulatory $\mathrm{T}$ cells and augmenting antitumor immunity. Nagoya J Med Sci 2019;81:1-18.

37. Zhou G, Drake CG, Levitsky HI. Amplification of tumorspecific regulatory $\mathrm{T}$ cells following therapeutic cancer vaccines. Blood 2006;107:628-36.

38. Ebert LM, MacRaild SE, Zanker D, et al. A cancer vaccine induces expansion of NY-ESO-1-specific regulatory T cells in patients with advanced melanoma. PLoS One 2012;7:e48424.

39. Noordam L, Kaijen MEH, Bezemer K, et al. Low-dose cyclophosphamide depletes circulating naïve and activated regulatory $T$ cells in malignant pleural mesothelioma patients synergistically treated with dendritic cell-based immunotherapy. Oncoimmunology 2018;7:e1474318.

40. Shimizu K, Nakata M, Hirami Y, et al. Tumorinfiltrating Foxp3+ regulatory $\mathrm{T}$ cells are correlated with cyclooxygenase- 2 expression and are associated with recurrence in resected non-small cell lung cancer. J Thorac Oncol 2010;5:585-90.

41. Zhang XW, Bi XW, Liu PP, et al. Expression of PD-L1 on Monocytes Is a Novel Predictor of Prognosis in Natural Killer/T-Cell Lymphoma. Front Oncol 2020;10:1360.

42. Gambichler T, Schröter U, Höxtermann S, et al. Decline of programmed death-1-positive circulating $T$ regulatory cells predicts more favourable clinical outcome of patients with melanoma under immune checkpoint blockade. Br J Dermatol 2020;182:1214-20.

Cite this article as: Zhang M, Hong JA, Kunst TF, Bond CD, Kenney CM, Warga CL, Yeray J, Lee MJ, Yuno A, Lee S, Miettinen M, Ripley RT, Hoang CD, Gnjatic S, Trepel JB, Schrump DS. Randomized phase II trial of a first-in-human cancer cell lysate vaccine in patients with thoracic malignancies. Transl Lung Cancer Res 2021;10(7):3079-3092. doi: 10.21037/ tlcr-21-1 
Table S1 Reagents used for this study

\begin{tabular}{|c|c|c|}
\hline \multicolumn{3}{|l|}{ Antibodies } \\
\hline Name & Company & Catalogue \# \\
\hline CT45 & United States Biological & 34313 \\
\hline NY-ESO-1 & Millipore Sigma & N2038 \\
\hline MAGE-A1 & AbCam & ab21472 \\
\hline MAGE-A3 & AbCam & ab38496 \\
\hline XAGE-1 & AbCam & ab134805 \\
\hline SSX1 & AbCam & ab206839 \\
\hline PRAME & AbCam & ab219650 \\
\hline BORIS & AbCam & ab187163 \\
\hline MHC Class I & AbCam & ab134189 \\
\hline b-Tubulin & AbCam & ab15568 \\
\hline CD45 & BioLegend & HI30 \\
\hline CD4 & BioLegend & RPA-T4 \\
\hline CD8 & BioLegend & SK1 \\
\hline CD25 & BioLegend & BC96 \\
\hline Foxp3 & BioLegend & $206 D$ \\
\hline CD14 & BioLegend & HCD14 \\
\hline CD16 & BioLegend & $3 G 8$ \\
\hline PD-1 & BioLegend & $29 \mathrm{~F}$ \\
\hline CTLA-4 & BioLegend & L3D10 \\
\hline TIM-3 & BioLegend & F38-2E2 \\
\hline HLA-DR & BioLegend & L243 \\
\hline PD-L1 & BioLegend & 29E.2A3 \\
\hline \multicolumn{3}{|c|}{ TaqMan primers and probes } \\
\hline Name & Company/sequence & Catalogue \# \\
\hline MAGE-A1 & Thermo Fisher & Hs00607097_m1 \\
\hline MAGE-A6 & Thermo Fisher & Hs00602508_m1 \\
\hline MAGE-A12 & Thermo Fisher & Hs04176236_m1 \\
\hline GAGE & Thermo Fisher & Hs00275620_m1 \\
\hline XAGE-1 & Thermo Fisher & Hs00220764_m1 \\
\hline SSX1 & Thermo Fisher & Hs00846692_s1 \\
\hline ssX2 & Thermo Fisher & Hs00817683_m1 \\
\hline CT45 F & \multicolumn{2}{|c|}{ 5'-СTCTGCCATGTCCAAAGCAA-3' } \\
\hline CT45 R & \multicolumn{2}{|c|}{ 5'-AAGTCATCAATCTGAGAATCCAATTG-3' } \\
\hline CT45 probe & \multicolumn{2}{|c|}{ 6FAM-AAGCTTATGACAGGACATGCTATTCCACCCA-TAMRA } \\
\hline NY_ESO-1 F & \multicolumn{2}{|c|}{ 5'-TGCTTGAGTTCTACCTCGCCAT-3' } \\
\hline NY-ESO-1 R & \multicolumn{2}{|c|}{ 5'-GCTCCTGCGGGCCAG-3' } \\
\hline NY-ESO-1 probe & \multicolumn{2}{|c|}{ 6FAM-TTTCGCGACACCCATGGAAGCAG-TAMRA } \\
\hline MAGE-A3 F & \multicolumn{2}{|c|}{ 5'-TCCTGTGATCTTCAGCAAAGCTT-3' } \\
\hline MAGE-A3 R & \multicolumn{2}{|c|}{ 5'-GGGTCCACTTCCATCAGCTC-3' } \\
\hline MAGE-A3 probe & \multicolumn{2}{|c|}{ 6FAM-CAGTTCCTTGCAGCTGGTCTTTGGCAT-TAMRA } \\
\hline BORIS F & \multicolumn{2}{|c|}{ 5'-CCCATTGTGCCACCATCA-3' } \\
\hline BORIS R & \multicolumn{2}{|c|}{ 5'-AGCATGCAAGTTGCGCATAT-3' } \\
\hline BORIS probe & \multicolumn{2}{|c|}{ 6FAM-TGCACGGAAAAGCGACCTACGTGTG-TAMRA } \\
\hline b-actin F & \multicolumn{2}{|c|}{ 5'-GCGAGAAGATGACCCAGATC-3' } \\
\hline b-actin $\mathrm{R}$ & \multicolumn{2}{|c|}{ 5'-CCAGTGGTACGGCCAGAGG-3' } \\
\hline b-actin probe & \multicolumn{2}{|c|}{ 6FAM-CCAGCCATGTACGTTGCTATCCAGGC-TAMRA } \\
\hline \multicolumn{3}{|l|}{ Cell lines } \\
\hline Name & Company & Catalogue \# \\
\hline H1299 & ATCC & CRL-5803 \\
\hline H841 & ATCC & CRL-5845 \\
\hline NHBE & Lonza & CC-2541 \\
\hline SAEC & Lonza & CC-2547S \\
\hline K562-GM & Barry & sity \\
\hline
\end{tabular}


A

$\%$ Tregs

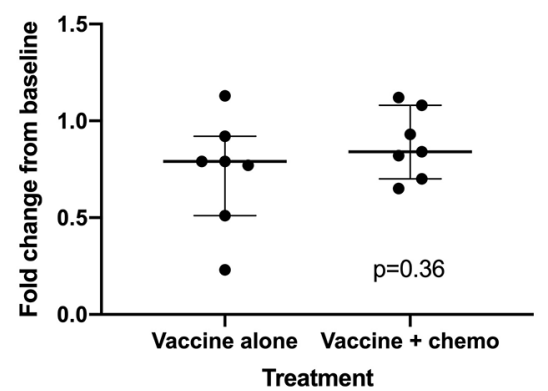

B

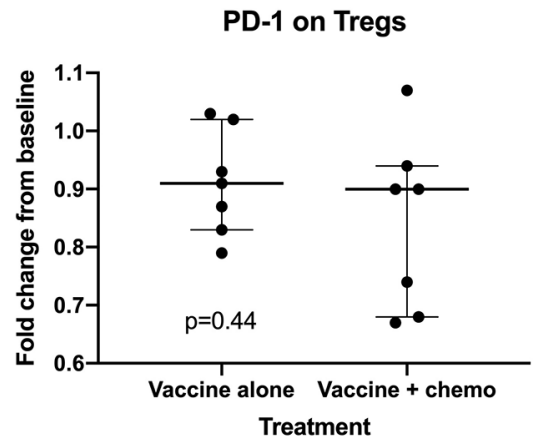

Figure S1 Comparison of fold change from baseline of percent Tregs (A) and intensity of PD-1 expression on Tregs (B) between vaccine alone and vaccine with chemotherapy. The Mann-Whitney $\mathrm{U}$ test was used to compare the two groups. 